
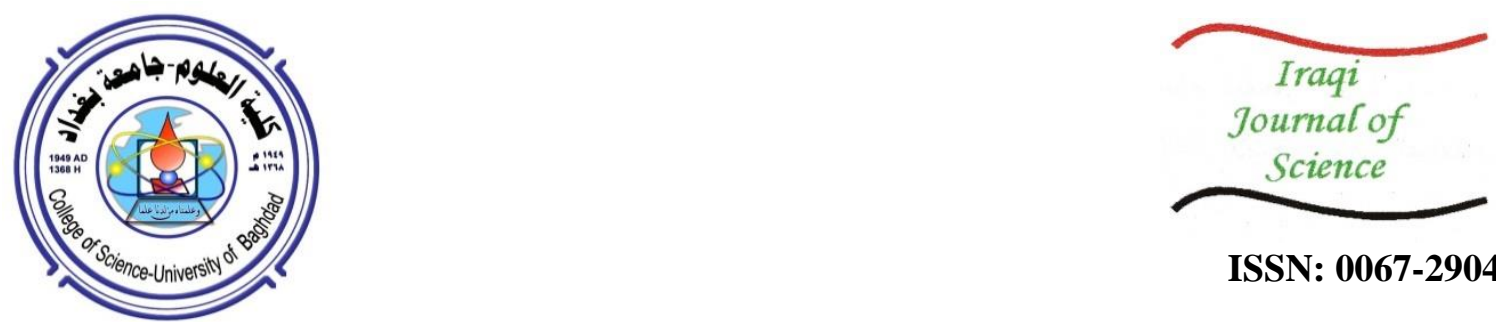

ISSN: 0067-2904

\title{
The Dependence of The Gravitational Lensing Properties on The Lens And Source Redshifts
}

\author{
Hiba Ahmed Abd Al-Lateef *, Hareth Saad Mahdi \\ Department of Astronomy and Space, College of Science, University of Baghdad, Baghdad, Iraq
}

Received: 27/5/2021

Accepted: 29/11/2021

\begin{abstract}
This work aims to investigate the dependence of gravitational lensing properties on the lens redshift and source redshift.

The angular diameter distance hereafter referred to as ADD has been determined using two different numerical integral methods, Simpson's rule, and definite integral methods. Both of those two methods gave identical results. In addition, observational data of gravitational Lensing systems have been used to find the most probable value of lens redshift and source redshift. The result showed that the lens redshift and source redshift are more likely to occur in the ranges of $z_{L}=0.2-0.6$ and $\mathrm{z}_{\mathrm{S}}=1-3$, respectively.

Einstein radius and the critical surface mass density have been studied for those ranges of lens and source redshift. The result clearly showed that the redshifts and distances affect the lensing properties. This indicates that observations of gravitational lensing can be efficiently used to probe the distances and redshifts.
\end{abstract}

Keywords: gravitational lensing, Redshift, angular diameter distance, critical surface mass density

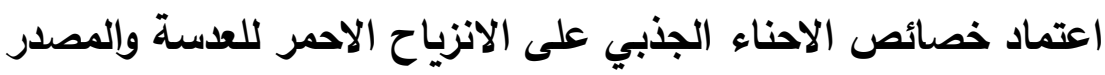




$$
\begin{aligned}
& \text { zL = أظهرت النتيجة أن الانزياح للعدسة والمصدر من المرجح أن يحدث ضمن هذه النطاقات على التوالي } \\
& \text {. } z_{S}=1-3,0.2-0.6 \\
& \text { تمت دراسة نصف قطر أينشتاين وكثافة كتلة السطح الحرجة لهذه النطاقات من الانزياحات الحمراء للعدسة }
\end{aligned}
$$

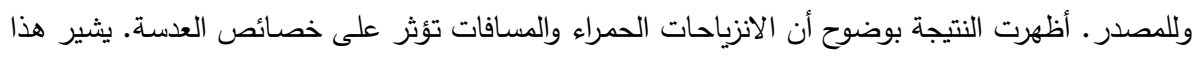

$$
\begin{aligned}
& \text { إلى أنه يمكن استخدام ملاحظات عدسة الجاذبية بكفاءة لاستكثاف المسافات والانزياحات الحمراء. }
\end{aligned}
$$

\section{Introduction}

In astronomy, an observer never sees an object's current state. However, the observer would be able to monitor the properties of the object's past. Observing distant objects provides us with information about the Universe's history, since we know our universe changes during time. Redshift increases with distance and time since light detected by an observer was emitted a time ago. Therefore, redshift $\mathrm{z}$ is commonly implemented in astronomical conceptions as a distance measure, and hence gives information about ages, and to specify time duration. Utilize redshift $\mathrm{z}$ holds an obvious feature which is a directly measured term, at least in this case the expression by $\mathrm{z}$ is exactly equivalent. [1].

Because the expansion of the Universe changes the distance between two commoving sources and an observer on Earth looks back in time as they lookout in the distance, there are different methods to characterize the distance between any two objects in the Universe.

The Angular Diameter Distance (ADD) is one of the most widely used cosmic distances, and it can be related to the comoving distance.

Gravitational Lensing (GL) is a unique probe in current astrophysics and one of the most important phenomena. [2]. It's one of Einstein's most famous predictions from his General Relativity (GR) theory, which he postulated in 1915. The existence of massive objects such as stars, black holes, galaxies, galaxy clusters distorts the fabric of space-time due to their huge masses, which means that large objects that travel fast across space-time, like galaxies or quasars, cause ripples in the fabric of space-time. [3]. Explanation of the realization of a double quasar (Q0957+561) in 1979 by Walsh has historical significance because it was the first multiply imaged quasar. The same cosmological redshift of two blue objects indicated that they belong to one object. The following finding out of several same orders confirmed that GL can make its way into the field of observational physics science [4]. Hence, nearly all present cosmological models are principled by (GR) theory that happened when gravity bent light act as natural telescopes that allow scientists to study faint and distant sources that would otherwise be beyond the reach of even the most powerful man-made telescopes, it means that the path of the photons from distance sources is twisted as light travels between source and observer, causing the sources' apparent size, shape, location, and magnitude to be affected by the foreground structure. 


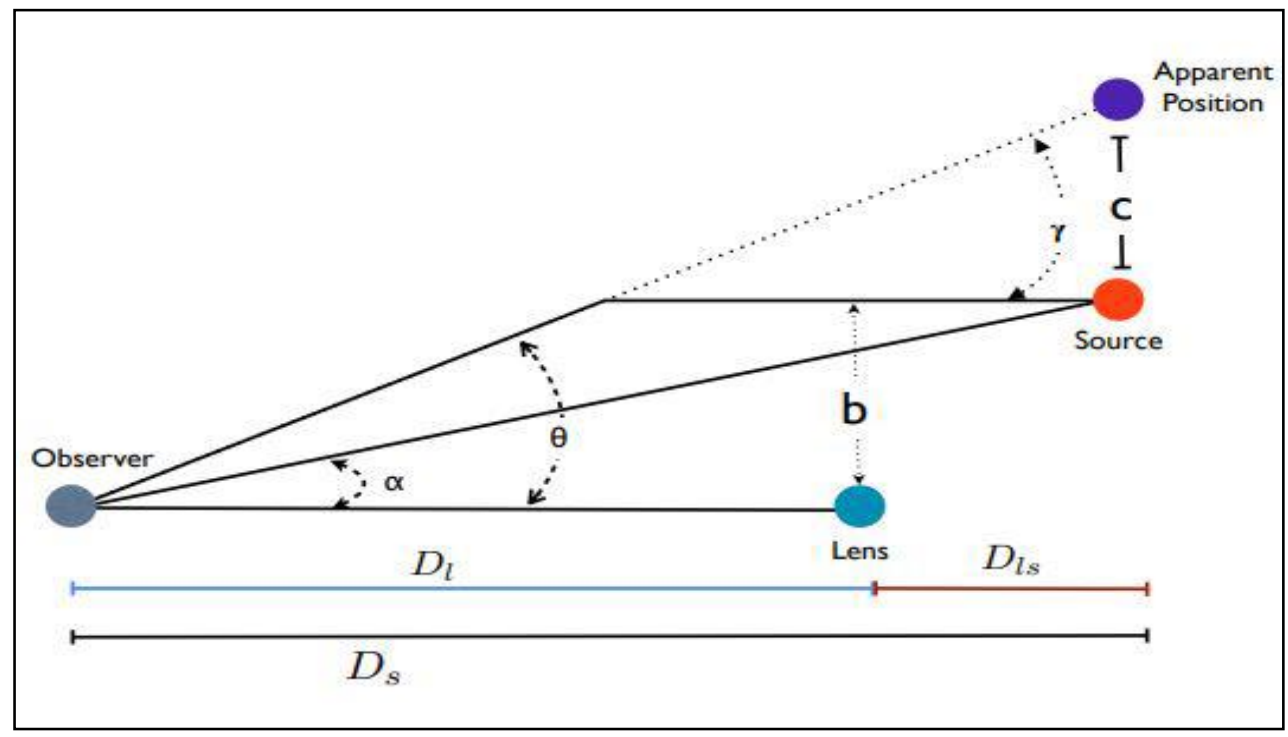

Figure 1-The geometry of gravitational lensing [5].

Figure 1 shows the geometry of gravitational lensing whereby the photons from the background source are deflected due to the existence of an intervening lens object, where $D_{L}$ is the distance to the lens, $D_{L S}$ is the distance between source and lens and $D_{s}$ is the distance from source to observer, note that for cosmological distances it is not in general true that $D_{S}=$ $\mathrm{D}_{\mathrm{L}}+\mathrm{D}_{\mathrm{LS}}$, despite the appearance in the diagram, assume that the lens can be approximated by a single object at defined distance $\mathrm{D}_{\mathrm{L}}$ the thin lens approximation and that all the angles are small.

Since gravitational lenses can magnify background sources, they have been used to study high redshift galaxies. In addition, lensing can turn a galaxy into an extended arc, making it possible to study its features in more details [5]. Strong lensing, weak lensing, and microlensing are the three types of gravitational lensing.

The concept of distance in curved space-times is more complicated; therefore, many distances are used in the gravitational lens effect. First, we consider the geometrical for the lens and source position. The distance between two celestial objects at the same redshift (distance) is known as transverse commoving distance. Therefore, they are expressed in coordinates referred to as commoving coordinates.

In the expanding Universe their many types of measurement distance. For example, the Angular Diameter Distance (ADD) is ratio of a source's physical size to its angular size. It is imperative because astronomers widely use it to obtain the proper separation from the angular separation in observed images [3]. In small distance, cosmological redshift is present by a linear relation. Still, large distance requires further knowledge about the expansion of the Universe as well as cosmological parameters.

GL has a wide range of applications in astrophysics, for example, in strong lensing, faint galaxies at high redshift can be used to detect if an intervening galaxy cluster is located in front of those galaxies. But, because these faint distant galaxies were formed early in the Universe, they cannot be detected without a magnifying foreground galaxy cluster.

The dependence of lensing signature on the properties of lens and source has been addressed by many previous works. For instance, a study by Meneghetti et al. (2007) used cosmological dark matter simulated clusters to probe the density profiles of galaxy clusters [6].

In addition, study of the Merging cluster A1758 by B. Ragozzine et al.(2012) that contains subclusters agree with previous weak-lensing results for clusters MACS J0025.4-1222 and Bullet cluster may provide information that would improve the mass measurements of A1758N [7], as well as the study of the strong lensing halos in massive galaxy clusters across redshift by Nan Li et al. (2019) that clarify the increase in strong lensing peak at different 
values of redshift gives information about the primary and secondary haloes for these massive galaxy clusters [8]

A recent study by fox et al.(2021) showed that the effective Einstein area correlates with lensing strength is an excellent result of a strong lens. The authors of this work pointed out that their findings could improve more efficient design of future observations to maximize lensing strength [9]. Formation and evaluation of galaxies and galaxy clusters become one of the key topics of modern Astronomy. Different methods are usually used to probe structures in the Universe. For instance, several studies were conducted recently to investigate chemical composition of galaxies( [10] and [11]). In addition, photometric studies of galaxies provide significant information about their physical properties (see, for example, [12] and [13]). Galaxy clusters, on the other hand, can be probed using their gravitational lensing signature (see [14], [15] and [16]).

The remainder of this paper is organized as follows: Next section presents the calculation and results, followed by the discussion and conclusions.

\section{Calculations and Results}

The goal of this study is to see how the characteristics of gravitational lensing are affected by redshift to the lens $z_{L}$ and redshift to the source $z_{S}$. A result led to the conclusion that gravitational lensing properties give information about the cosmos structure.

Then ADD is calculated from the following formula [17]:

$$
D_{A}=\frac{D_{M}}{1+z}
$$

where $\mathrm{z}$ is the redshift and $\mathrm{D}_{\mathrm{M}}$ is the transverse comoving distance, the comoving distance between two events at the same redshift or distance is simply related to the line-of-sight comoving distance. In a flat universe for which the curvature density parameter $\left(\Omega_{K}=0\right)$, then ADD is associated to $\mathrm{D}_{M}$ via [17]:

$$
D_{M}=D_{C} \quad \text { for } \Omega_{K}=0
$$

where $D_{c}$ is the commoving distance, which is given by the following formula [17]:

$$
\mathrm{D}_{\mathrm{c}}=\mathrm{D}_{\mathrm{H}} \int_{0}^{z} \frac{d z}{\sqrt{\Omega_{M}(1+z)^{3}+\Omega_{\Lambda}}} \text { for } \Omega_{\mathrm{k}}=0
$$

While $\mathrm{D}_{\mathrm{H}}$ is known as the Hubble distance and is given by [17]:

$$
\mathrm{D}_{\mathrm{H}} \equiv \frac{\mathrm{c}}{\mathrm{H}_{0}}
$$

Where $\mathrm{c}$ is the speed of light and $\mathrm{H}_{0}$ is Hubbel's constant.

Therefore, equation (1) becomes:

$$
\mathrm{D}_{\mathrm{A}}=\frac{\mathrm{D}_{\mathrm{H}} \int_{0}^{\mathrm{z}} \frac{\mathrm{dz}}{\sqrt{\Omega_{\mathrm{M}}(1+\mathrm{z})^{3}+\Omega_{\Lambda}}}}{1+\mathrm{z}}
$$

(5) The angular diameter distance $\mathrm{D}_{\mathrm{LS}}$ between the lens and source in gravitational lensing is given by:

$$
\mathrm{D}_{\mathrm{LS}}=\frac{1}{1+\mathrm{z}_{\mathrm{S}}}\left[\mathrm{D}_{\mathrm{MS}}-\mathrm{D}_{\mathrm{ML}}\right] \text { For } \Omega_{\mathrm{k}}=0
$$

where $\mathrm{D}_{\mathrm{MS}}$ and $\mathrm{D}_{\mathrm{ML}}$ are the transverse comoving distances for $\mathrm{z}_{\mathrm{L}}$ and $\mathrm{z}_{\mathrm{S}}$ [17].

In this research, the (ADD) from Eq. (5) has been determined using two different integral methods; the Simpson's rule and the definite integral method.

A significant purpose in numerical integration is to achieve precise results with a limited number of functions evaluations. Numerical integration ways approximate a definite integral by evaluating the integrand at many points and taking a weighted summation of those integrand values [18]. Simpson's rule and the Definite Integral seem to be the most widely used procedure to calculate the area under the curve.

Simpson's rule can be obtained from Newton-Cote's formulas, which represents an approximation of $\int_{a}^{b} \mathrm{f}(\mathrm{x}) \mathrm{dx}$. 
To get combined Simpson's rule, the integration range (a-b) is divided into (n-1) panels (n odd) [19]:

$$
\int_{a}^{b} f(x) d x \approx I=\left[f\left(x_{1}\right)+4 f\left(x_{2}\right)+2 f\left(x_{3}\right)+4 f\left(x_{4}\right)+\cdots\right.
$$

Simpson's rule demands the number of panels to be even if this condition is not satisfied we can integrate over the first or last three panels with Simpson's 3/8 rule:

Where $h$ is $h=(b-a) /(n-1)$.

$$
I=\left[f\left(x_{1}\right)+3 f\left(x_{2}\right)+3 f\left(x_{3}\right)+f\left(x_{4}\right)\right] \frac{3 h}{8}
$$

Dividing the interval (a-b) into many parts by shifting integration limits might be exploited to esteem the contribution from each interval.

The definite integral method is commonly used to determine if the area under a curve is not specified, but rather the limit of the summation, as the basis for making this definition. It shows how to calculate approximations to such a region. We can obtain an approximation value for the area by using a big worth of $n$ and determining upper and lower sums. The difference between the upper and lower sum can be written as the form below:

$$
I=\lim _{n \rightarrow \infty} \sum_{i=1}^{n} f\left(x_{i}^{*}\right) \Delta \mathrm{x}
$$

We denote that $\int_{a}^{b} \mathrm{f}(\mathrm{x}) \mathrm{dx}$, where (a-b) is already divided into (n) equal subintervals, every with a width of $\Delta \mathrm{x}$, while $\mathrm{x}_{i}$ is a point in the $i$ th subinterval.

The distinction is that it refers to the limit of a sum instead of a finite sum in this situation. As we approach the limit, the $\mathrm{dx}$ emanates from the $\Delta \mathrm{x}$ as a result. The definite integral is defined as a border to a specific form of total, such as in Eq. (9) above, as the number of subintervals approaches infinity and the width of each subinterval approaches (zero). [20]

The angular diameter distance can be calculated by two above methods for redshift in the range of (0-5), and relation between ADD and $\mathrm{z}$ is shown in Figure 2.

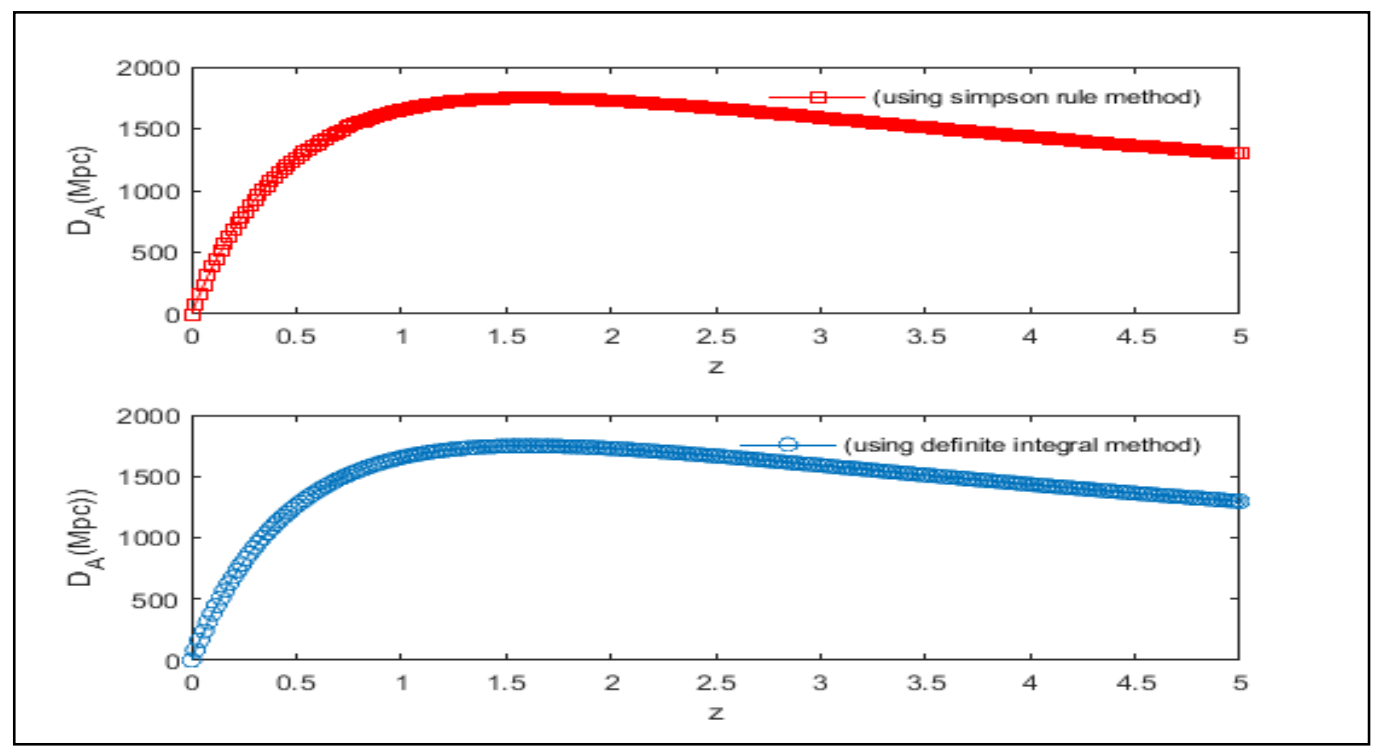

Figure 2 -The relation between ADD and redshift

To obtain the most probable values of lens redshift $\mathrm{z}_{\mathrm{L}}$ and source redshift $\mathrm{z}_{\mathrm{S}}$, observational data of gravitational lensing systems have been collected from the literature [21] [22] [23], and a sub-sample of those observations is listed in Table 1: 
Table 1- A sub-sample of the gravitational lensing data [21] [22] [23].

\begin{tabular}{|c|ccc|c|ccc|}
\hline No. & source name & $\mathbf{z}_{\mathbf{L}}$ & $\mathbf{z}_{\mathbf{S}}$ & No. & source name & $\mathbf{z}_{\mathbf{L}}$ & $\mathbf{z}_{\mathbf{S}}$ \\
\hline $\mathbf{1}$ & B0850+054 & 0.59 & 1.14 & $\mathbf{2 1}$ & QJ01584325 & 0.317 & 1.29 \\
$\mathbf{2}$ & B1030+074 & 0.6 & 1.54 & $\mathbf{2 2}$ & RXJ0921+4529 & 0.31 & 1.65 \\
$\mathbf{3}$ & B1608+656 & 0.63 & 1.39 & $\mathbf{2 3}$ & RXJ11311231 & 0.295 & 0.658 \\
$\mathbf{4}$ & B2114+022 & 0.32 & 0.59 & $\mathbf{2 4}$ & RXJ1131-1231 & 0.295 & 0.658 \\
$\mathbf{5}$ & BRI09520115 & 0.632 & 4.5 & $\mathbf{2 5}$ & SDSS1155+6346 & 0.176 & 2.89 \\
$\mathbf{6}$ & CFRS03.1077 & 0.938 & 2.941 & $\mathbf{2 6}$ & SDSS1332+0347 & 0.191 & 1.445 \\
$\mathbf{7}$ & CY22013201 & 0.32 & 3.9 & $\mathbf{2 7}$ & SDSS1353+1138 & 0.3 & 1.629 \\
$\mathbf{8}$ & MG0414+0534 & 0.96 & 2.64 & $\mathbf{2 8}$ & SDSS1402+6321 & 0.2 & 0.48 \\
$\mathbf{9}$ & FBQ0951+2635 & 0.24 & 1.24 & $\mathbf{2 9}$ & SDSS1406+6126 & 0.27 & 2.13 \\
$\mathbf{1 0}$ & FSC10214+4724 & 0.75 & 2.29 & $\mathbf{3 0}$ & SDSSJ002907.8005550 & 0.227 & 0.931 \\
$\mathbf{1 1}$ & HE02302130 & 0.52 & 2.162 & $\mathbf{3 1}$ & SDSSJ0157558.9005626 & 0.513 & 0.924 \\
$\mathbf{1 2}$ & HE0512-3329 & 0.93 & 1.57 & $\mathbf{3 2}$ & SDSSJ021652.5081345 & 0.332 & 0.523 \\
$\mathbf{1 3}$ & J1004+1229 & 0.95 & 2.65 & $\mathbf{3 3}$ & SDSSJ025245.2+003958 & 0.28 & 0.982 \\
$\mathbf{1 4}$ & MG1549+3047 & 0.11 & 1.17 & $\mathbf{3 4}$ & SDSSJ033012.1002052 & 0.351 & 1.107 \\
$\mathbf{1 5}$ & MG1654+1346 & 0.25 & 1.74 & $\mathbf{3 5}$ & SDSSJ072805.0+383526 & 0.206 & 0.688 \\
$\mathbf{1 6}$ & Q1017-207 & 0.78 & 2.55 & $\mathbf{3 6}$ & SDSSJ090315.2+411609 & 0.43 & 1.065 \\
$\mathbf{1 7}$ & Q13552257 & 0.48 & 1.37 & $\mathbf{3 7}$ & SDSSJ091205.3+002901 & 0.164 & 0.324 \\
$\mathbf{1 8}$ & Q1355-2257 & 0.48 & 1.37 & $\mathbf{3 8}$ & SDSSJ120540.4+491029 & 0.215 & 0.481 \\
$\mathbf{1 9}$ & Q0957+561 & 0.36 & 1.41 & $\mathbf{3 9}$ & SDSSJ142015.9+601915 & 0.063 & 0.535 \\
$\mathbf{2 0}$ & Q2237+030 & 0.04 & 1.69 & $\mathbf{4 0}$ & WFI20334723 & 0.66 & 1.66 \\
\hline
\end{tabular}

The data of lens and source redshifts have been used to produce histogram. The histograms of lens redshift and source redshift are shown in Figure 3. It shows that lens redshift is more likely to occur in $\left(\mathrm{z}_{\mathrm{L}}=0.2\right.$ to 0.6$)$, while source redshift is more likely to happen in the range $\left(\mathrm{z}_{\mathrm{S}}=1\right.$ to 3$)$.

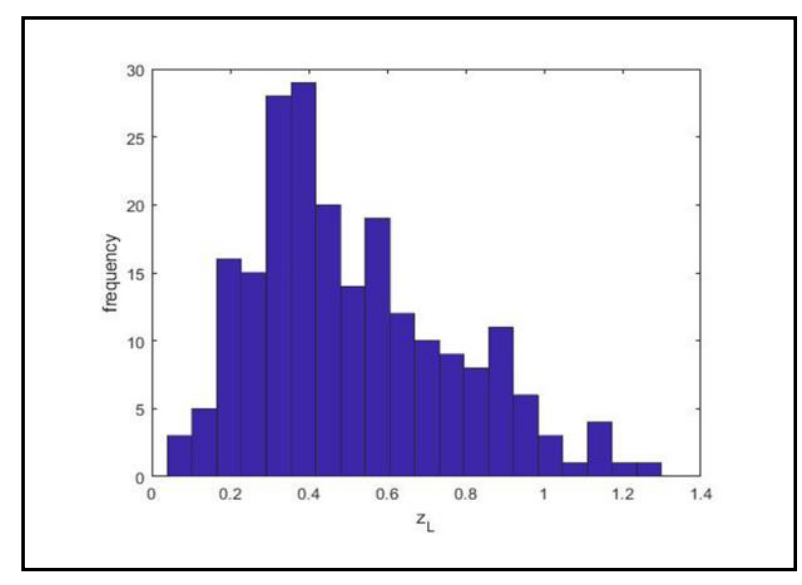

(a)

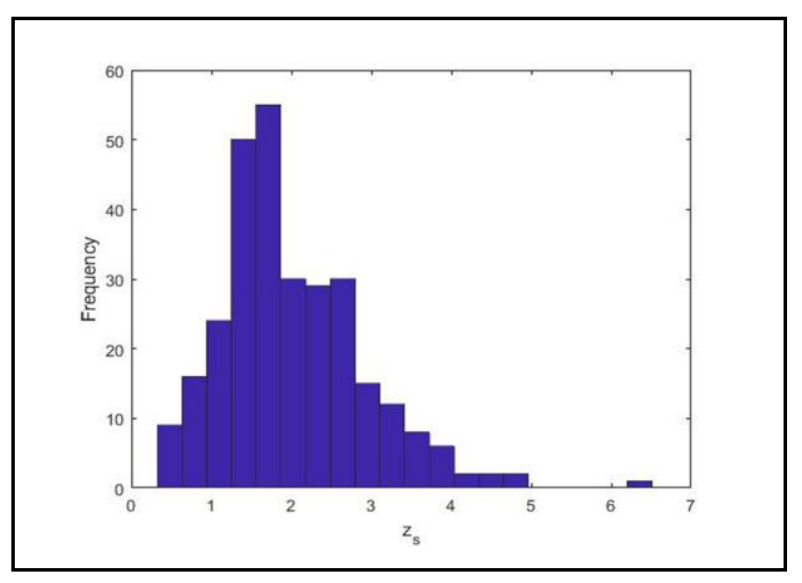

(b)

Figure 3-The figure shows the histogram of the lens redshift (a) and source redshift (b).

Therefore, these redshift ranges have been used in this work to study the lensing properties of galaxy clusters with a mass of $10^{14} \mathrm{M}_{\odot}\left(\mathrm{M}_{\odot}\right.$ : is the solar mass). Particularly, the dependence of Einstein radius $\theta_{E}$ and the critical surface mass density $\Sigma_{c r}$ On the lens redshift and source, redshift has been investigated.

In gravitational lensing, a ring-like image occurs if the mass distribution of the lens is spherical and the observer-lens-source is co-aligned. The radius of this image is called Einstein radius $\theta_{E}$ and is given by the equation below [2]:

$$
\theta_{E}=\sqrt{\frac{4 G M}{c^{2}} \frac{D_{L S}}{D_{L} D_{S}}}
$$


Einstein radius is a very important concept because it supplies a scale for surface mass density $\Sigma$. Due to Eq. (1), the area within $\theta_{E}$ Itself proportional to the mass. the critical surface mass density $\Sigma_{c r}$ the equation is given below [2]:

$$
\Sigma_{c r}=\frac{c^{2}}{4 \pi G} \frac{D_{S}}{D_{L} D_{L s}}
$$

where $\mathrm{c}$ is the speed of light whose value is $\left(3 \times 10^{8} \mathrm{~m} / \mathrm{s}\right)$, and $(\mathrm{G})$ is the universal gravitational constant $\left(6.67 \times 10^{-11} \mathrm{~m}^{3} \cdot \mathrm{kg}^{-1} \cdot \mathrm{s}^{-2}\right)$.

Einstein radius and critical surface mass density have been determined for lens redshift in the range of $\mathrm{z}_{\mathrm{L}}=0.2-0.6$ considering three different values of source redshift $\mathrm{z}_{\mathrm{S}=1} 1,2$, and 3 . Einstein radius as well as the $\Sigma_{c r}$ as a function lens redshift are shown in Figure 4.

The results showed that at each value of $z_{L}$, Einstein radius increase with redshift to the source $\mathrm{z}_{\mathrm{S}}$ as shown in the upper panel of the figure 4 , and $\Sigma_{c r}$ decreases as shown in the lower panel of the Figure 4.
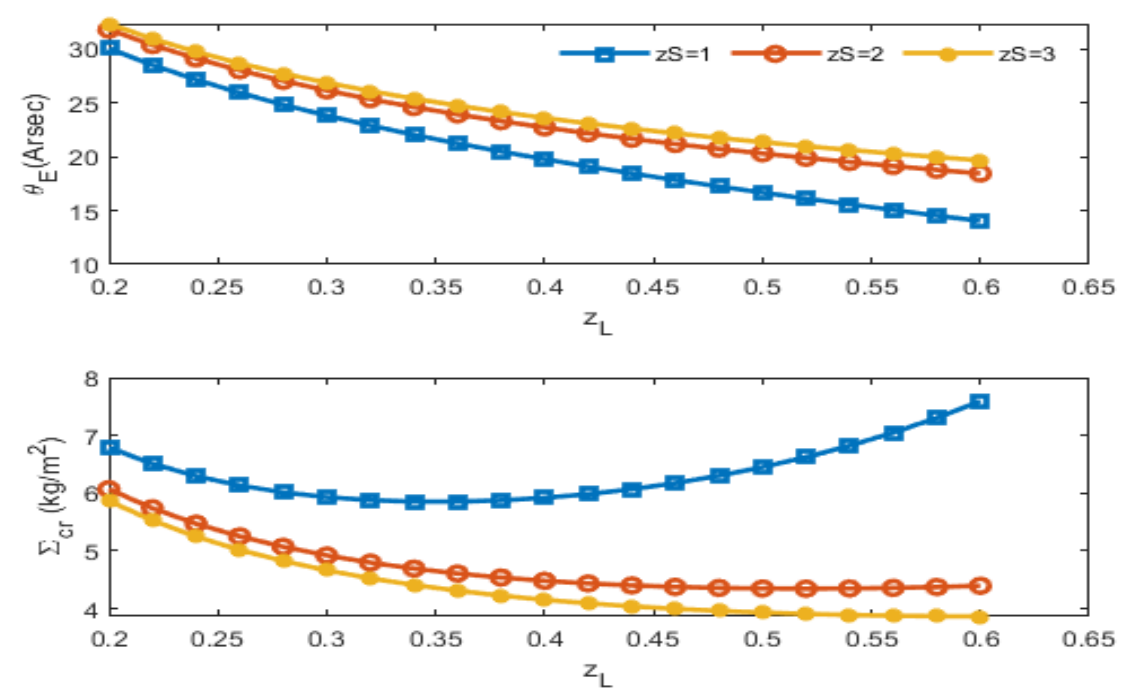

Figure 4-Einstein radius as a function of lens redshift (upper panel) and the critical surface mass density as a function of lens redshift (lower panel)

Then Einstein radius and critical surface mass density have been determined for source redshift in the range of $\mathrm{z}_{\mathrm{S}}=1-3$ considering three different values of lens redshift $\mathrm{z}_{\mathrm{L}}=0.2,0.4$ and 0.6. Einstein radius is versus source redshift and $\Sigma_{c r}$ versus source redshift shown in Figure 4. From this figure, one can notice that at each value of $z_{S}$, Einstein radius decreases with redshift to the Lens $z_{L}$ as shown in the upper panel of the figure -5 and $\Sigma_{c r}$ decreases with redshift to source $z_{S}$ as shown in the lower panel in Figure 5. 


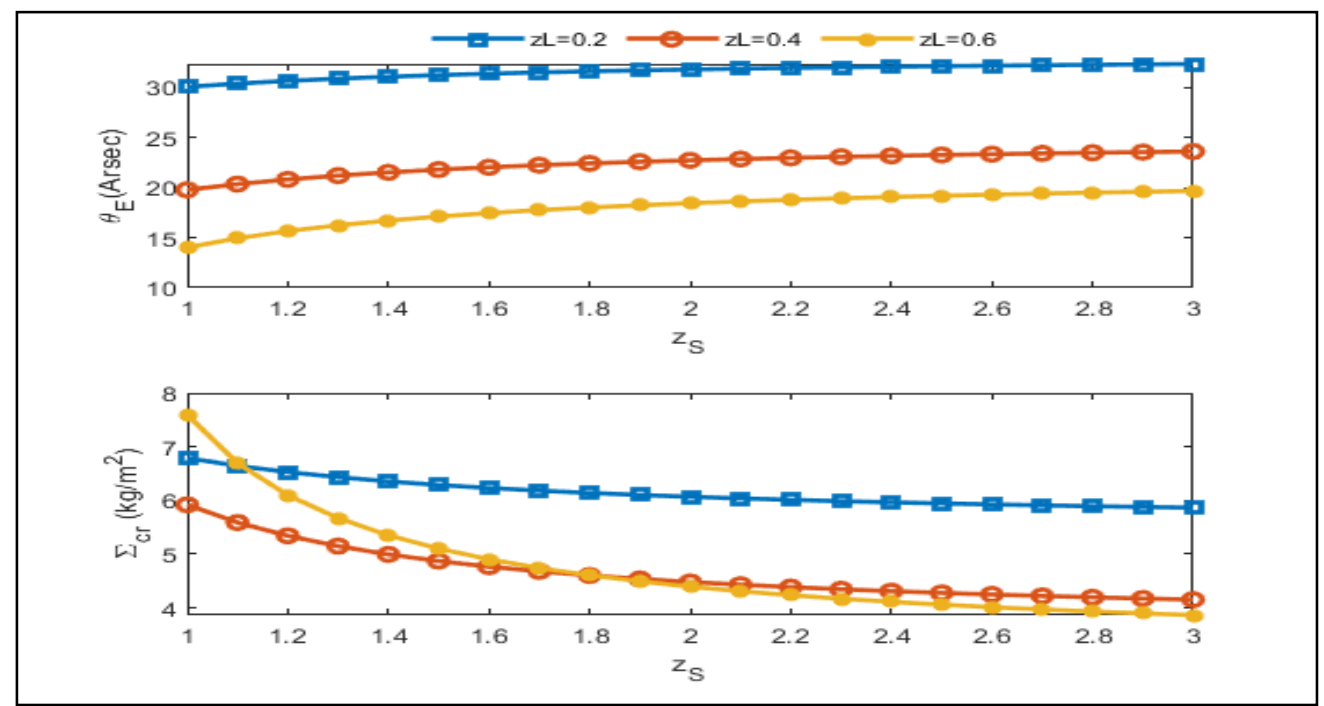

Figure 5-Einstein radius as a function of $\mathrm{z}_{\mathrm{S}}$ (upper panel) and $\Sigma_{c r}$ as a function of $\mathrm{z}_{\mathrm{S}}$ (lower panel).

The behaviour shown in Figure 4 is due to the effect on ADD between lens and source, observer and source, and observer and lens on $\theta_{E}$ and $\Sigma_{c r}$ as shown in Figure 6. Angular diameter distance $D_{L}, D_{L s}$, and $D_{s}$ have been plotted as a function of lens redshift of $z_{L}$ in the range of (0.2-0.6).

The result clearly showed that at each value of $z_{L}, D_{L}$ remains constant. In addition the values of $D_{L S}$ at $z_{S}=2$ and 3 are higher than those at $z_{S}=1$, while the values of $D_{L S}$ at $z_{S}=3$ are smaller than $z_{S}=2$. This is because the ADD decreases at higher redshift as explain in Figure 2 . Furthermore, $D_{S}$ at $z_{S}=2$ is higher than that at $z_{S}=1$ and 3 . This is again due to the effect of the expansion of the Universe.

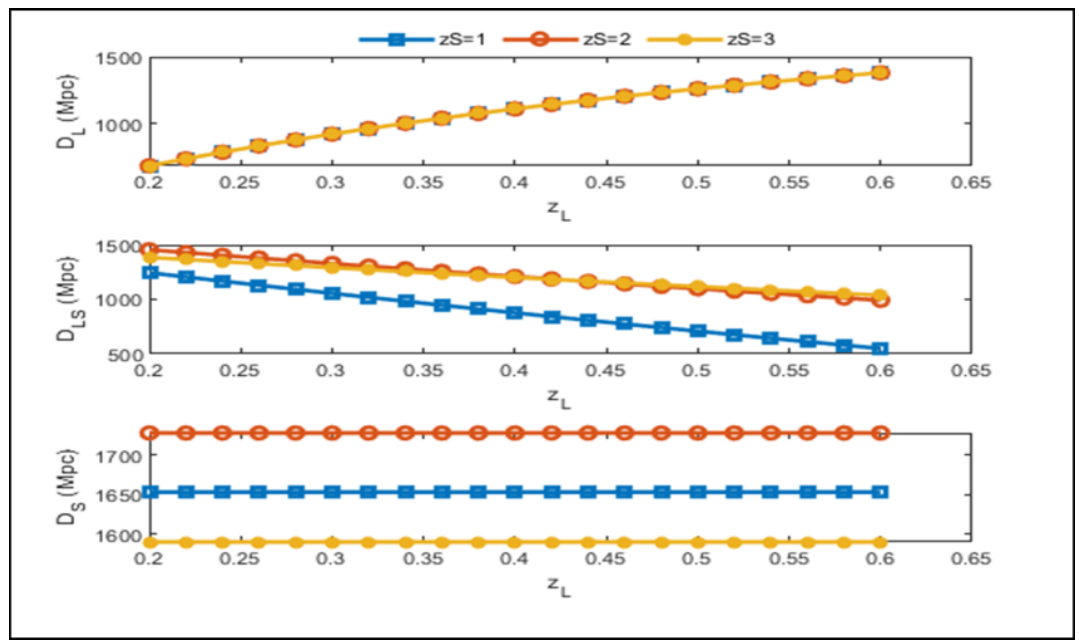

Figure -6 The distances $D_{L}, D_{L S}$ and Ds as a function of lens redshift

Figure 7 explains the relations between $\Sigma_{c r}$ and $\left(\mathrm{D}_{\mathrm{S}} / \mathrm{D}_{\mathrm{LS}}\right)$ as well as $\theta_{E}$ and $\left(\mathrm{D}_{\mathrm{LS}} / \mathrm{D}_{\mathrm{S}}\right)^{1 / 2}$. From the upper panel, one can notice that $\left(\mathrm{D}_{\mathrm{S}} / \mathrm{D}_{\mathrm{LS}}\right)$ is directly proportional to the $\mathrm{z}_{\mathrm{S}}$, while the lower panel shows that $\left(\mathrm{D}_{\mathrm{LS}} / \mathrm{D}_{\mathrm{S}}\right)^{1 / 2}$ is inversely proportional to $\mathrm{z}_{\mathrm{S}}$. 


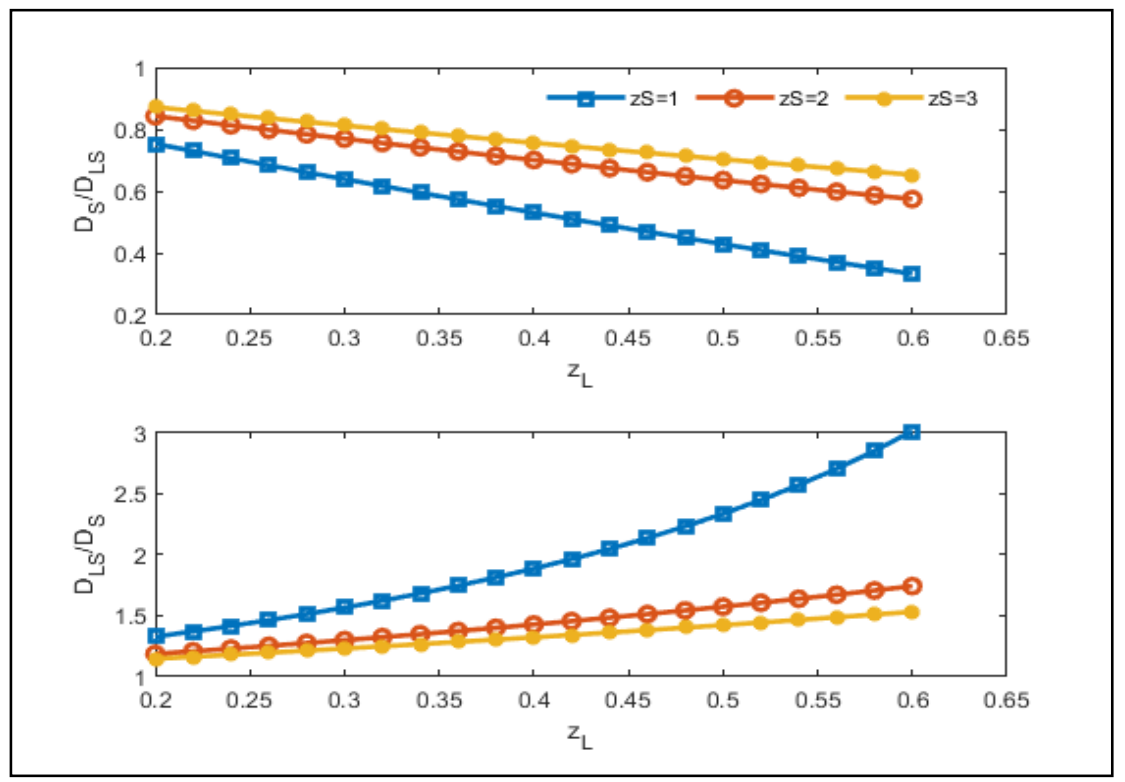

Figure -7 $\left(\mathrm{D}_{\mathrm{S}} / \mathrm{D}_{\mathrm{LS}}\right)$ and $\left(\mathrm{D}_{\mathrm{LS}} / \mathrm{D}_{\mathrm{S}}\right)^{1 / 2}$ versus lens redshift $\mathrm{z}_{\mathrm{L}}$

In Figure $8, D_{L}, D_{L S}$, and $D_{S}$ have been plotted against the source redshift. The results showed that at each value of $z_{S}, D_{L}$ increases with redshift to the lens, while $D_{L S}$ decreases and $D_{S}$ remains constant.

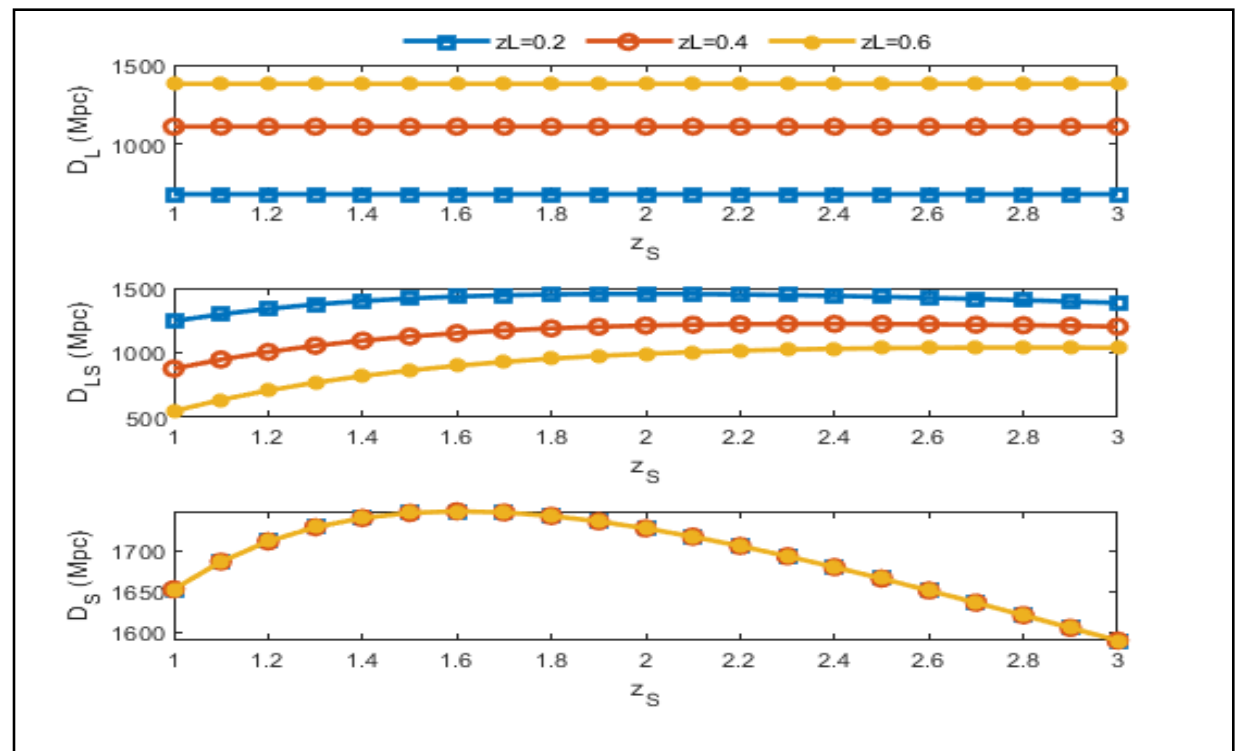

Figure 8 -The distances $\left(D_{L}, D_{L S}, D_{S}\right)$ as a function of redshift $z_{S}$

Therefore, Einstein radius is proportional to $\left(D_{L S} / D_{L}\right)^{1 / 2}$ with redshift $z_{S}$ as shown in the upper panel of Figure 9, while $\Sigma_{c r}$ is proportional to $\left(1 / \mathrm{D}_{\mathrm{L}} \mathrm{D}_{\mathrm{LS}}\right)$ as illustrated in the lower panel of Figure 9. This indicates that the change in $\theta_{E}$ due to the change in $\left(D_{L S} / D_{L}\right)^{1 / 2}$ and the change in $\Sigma_{c r}$ is attributed to the change in $\left(1 / \mathrm{D}_{\mathrm{L}} \mathrm{D}_{\mathrm{LS}}\right)$ as one can notice in Eq. (10) and Eq. (11). 

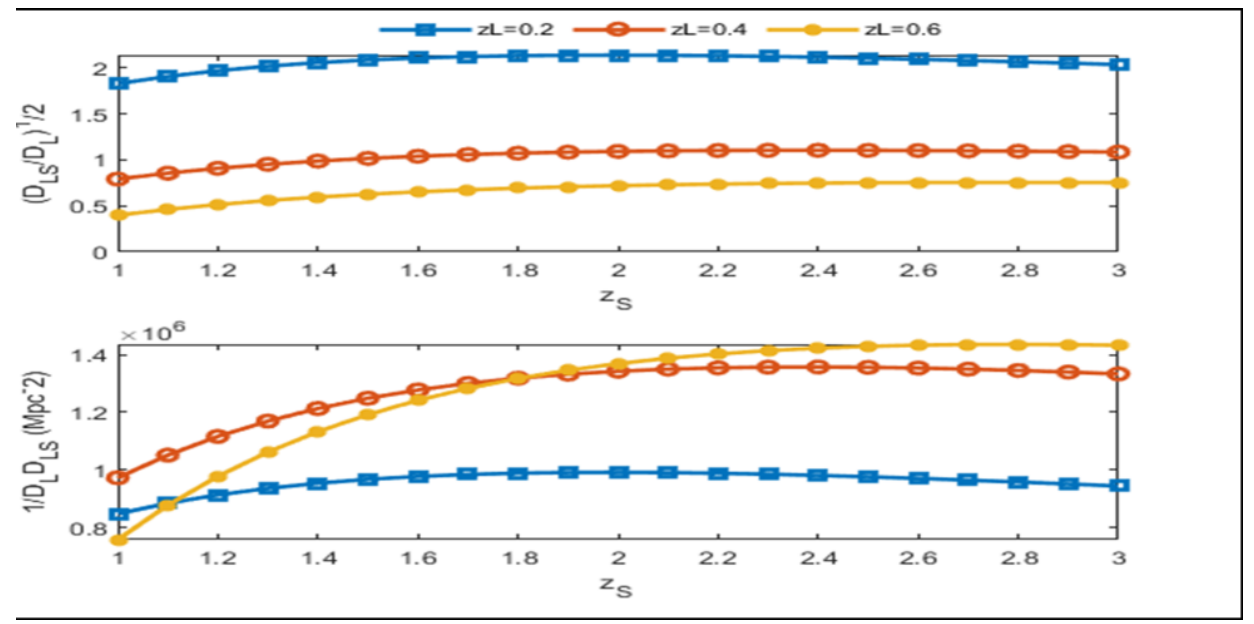

Figure -9 The ratio of $\left(\mathrm{D}_{\mathrm{LS}} / \mathrm{D}_{\mathrm{L}}\right)^{1 / 2}$ and $\left(1 / \mathrm{D}_{\mathrm{L}} \mathrm{D}_{\mathrm{LS}}\right)\left(\mathrm{Mpc}^{-2}\right)$ behavior versus source redshift.

\section{Discussion and Conclusions}

This research demonstrates that the ADD is dependent on the Universe's geometry as well as its cosmological parameters. As a result, gravitational lensing observations can reveal information about the Universe's distance and shape. This provides precise information for studying galaxies and galaxy clusters in the Universe. The data revealed that ADD increases with redshift until it reaches a certain point, beyond which it begins to decline; this is because the expansion of the Universe was smaller in the past, resulting in a shorter distance.

At higher redshift, the angular diameter distance was characterized by a low rate because space-time was compressed when the light was emitted, the galaxies were closer to us than they are today. In the current time, the distances are changed because of the expansion that happened in our Universe that was discovered from the changed redshifts. The concept of redshift is very important to investigate our expanding Universe.

Observation data of gravitational lensing systems have been collected from public databases and literature. It has been noticed that the lens redshift is more likely to occur in the range of 0.2 to 0.4 . Therefore, we have studied the dependence of Einstein radius and critical surface mass density on the lens redshift and source redshift with those ranges.

The results demonstrated that the lensing properties such as Einstein radius and critical surface mass density were affected by angular diameter distances. Hence, gravitational lensing observational data may be used to constrain the distances between the observer and the source, the observer and the lens, and the lens and the source.

\section{Reference}

[1] I. Appenzeller, High redshift galaxies, Germany: Springer, 2009.

[2] J. Wambsganss, "Gravitational Lensing in Astronomy," Living Rev. Relativ, p. 49, 1121998.

[3] M. Moti, The Relativity on theory, Melbourne, FL, USA: Springer, 2020.

[4] B. Congdon and C. R. Ketoon, Principles of Gravitational lensing, Piscataway, NJ, USA: Springer Nature Switzerland, 2018.

[5] J. Young, B. S and M. S, Improving accuracy in gravitational weak lensing measurements of clusters, Ohio: The Ohio State University, 2013.

[6] M. Meneghetti and e. al., "The effects of ellipticity and substructure on estimates of cluster density profiles based on lensing and kinematics," vol. 381, no. 1, p. 16, 2007.

[7] B. Ragozzine and e. al., "WEAK-LENSING RESULTS FOR THE MERGING CLUSTER A1758," The Astrophysical Journal, vol. 744, no. 2, p. 9, 2012. 
[8] N. Li, M. D. Gladders and e. a. , "The Importance of Secondary Halos for Strong Lensing in Massive Galaxy Clusters across Redshift," The Astrophysical Journal, vol. 878, no. 2, p. 10, 2019.

[9] C. Fox, "The Strongest Cluster Lenses: An Analysis of the Relation Between Strong Gravitational Lensing Strength and the Physical Properties of Galaxy Clusters," arXiv:2104.05585v1, p. 31, 2021.

[10] M. N. ALNajm, H. S. Mahdi and S. A. Abdullah, "The Exponential and Gaussian Density Profiles of HI and Fe II in the Gaseous Halo of the Milky Way," Iraqi Journal of Science, vol. 58, no. 4C,pp:2467-2472, p. 6, 2017.

[11] M. N. AL Najm, "Studying the Atomic and Molecular Hydrogen Mass (MHI, MH2)," Iraqi Journal of Science, vol. 61, no. 5,pp:1233-1243, p. 11, 2020.

[12] H. R. AL-baqir, A. K. Ahmed and D. Jamal, "Surface Photometry of NGC 3 Lenticular Galaxy," Iraqi Journal of Science, vol. 60, no. 9,pp: 2080-2086, p. 7, 2019.

[13] Z. Adnan and A. K. Ahmed, "Photometric investigations of NGC 2577 and NGC 4310 Lenticular Galaxies," Iraqi Journal of Science, vol. 58, no. 2C, pp: 1129-1138, p. 10, 2018.

[14] P. J. Elahi, H. S. Mahdi, C. Power and G. F. Lewis, "Warm dark haloes accretion histories and their gravitational signatures," Monthly Notices of the Royal Astronomical Society, vol. 444, no. 3, p. 13, 2014.

[15] H. S. Mahdi, M. v. Beek, P. J. Elahi, G. F. Lewis, C. Powe and M. Killedar, "Gravitational lensing in WDM cosmologies: the cross-section for giant arcs," Monthly Notices of the Royal Astronomical Society, vol. 441, no. 3, p. 10, 2014.

[16] H. S. Mahdi, P. J. Elahi, G. F. Lewis and C. Power, "MATTER IN THE BEAM: WEAK LENSING, SUBSTRUCTURES, AND THE TEMPERATURE OF DARK MATTER," The Astrophysical Journal, vol. 826, p. 6, 2016.

[17] D. W. Hogg, "Distance measures in cosmology," astro-ph/9905116v4, p. 16, 2000.

[18] H. B. Wilson, L. H. Turcotte and D. Halpern, Advanced mathematics and mechanics applications using MATLAB, United States of America: Chapman \& Hall/CRC, 2003.

[19] J. Kiusalaas, Numerical Methods in Engineering with MATLAB, United States of America: Cambridge University Press, 2005.

[20] M. Barnes, "Introduction to Integration Part 2: The Definite Integral," University of Sydney, 1999. [Online]. Available: https://www.sydney.edu.au/content/dam /students/documents /mathematics-learning-centre/integration-definite-integral.pdf.

[21] R. Gavazzi, T. Treu, J. Rhodes, L. Koopmans, A. Bolton, S. Burles, R. Massey and L. Moustakas, "The Sloan Lens ACS Survey.IV: the mass density profile of early-type galaxies out to 100 effective radii.," The Astrophysical journal, vol. 667, no. 1, 2007.

[22] "Gravitationally Lensed Quasar Database," research.ast.cam.ac.uk, [Online]. Available: https://research.ast.cam.ac.uk/lensedquasars/.

[23] C. S. Kochanek, E. E. Falco, C. Impey, J. Lehar and B. Mcleod, "CASTLES Survey," [Online]. Available: https://www.cfa.harvard.edu/castles/. 